\title{
PLANTAS ÚTILES EN LOS SISTEMAS AGROFORESTALES TRADICIONALES DEL LITORAL ECUATORIANO
}

\author{
Luz García Cruzatty ${ }^{1}$, Pedro Suatunce Cunuhay ${ }^{1,2}$ y Emma Torres Navarrete ${ }^{3}$ \\ ${ }^{1}$ División Forestal, Unidad de Investigación Científica y Tecnológica, Universidad Técnica Estatal de Quevedo. Av. Walter \\ Andrade. Km 11/2 vía a Santo Domingo, C.P. 73. Quevedo, Los Ríos, Ecuador. luzcecilia29@hotmail.com. \\ ${ }^{2}$ Escuela de Ingeniería Forestal, Facultad de Ciencias Ambientales, Universidad Técnica Estatal de Quevedo. \\ ${ }^{3}$ División Agrosocioeconómica, Unidad de Investigación Científica y Tecnológica, Universidad Técnica Estatal de Quevedo.
}

\begin{abstract}
RESUMEN
El estudio se realizó en la Cuenca Media del Río Guayas, Ecuador, en el área correspondiente a los cantones Mocache y Palenque. El inventario de las especies se obtuvo mediante recorridos participativos. Las especies se clasificaron de acuerdo al Sistema Filogenético de Cronquist. Los datos sobre el uso de las especies se obtuvieron mediante charlas y entrevistas no formales. Se determinó que los agricultores de la Cuenca Media del Río Guayas conocen y utilizan 262 especies vegetales que pertenecen a 67 familias y 164 géneros. Las familias vegetales más comunes fueron: Anacardiaceae, Papilonaceae, Solanaceae, Rutaceae, Musaceae y Lamiaceae, con más de diez especies cada una. Se documentaron 21 tipos de usos, de los cuales los más importantes son: alimenticio, medicinal y construcción, lo que demuestra la preocupación de las familias por satisfacer sus necesidades básicas. La mayoría de las especies utilizadas son árboles, seguido por hierbas y arbustos. Entre las especies de mayor importancia por los múltiples usos y productos que se obtienen están: Persea americana Mill. (aguacate), Artocarpus altilis Fosberg (fruta de pan), Musa sp. (plátano), Manguifera $s p$. (mango), Phythelephas aequatoriales Spruce (cade), Inga edulis Matius (guaba de bejuco), entre otros.
\end{abstract}

Palabras clave: Uso tradicional, etnobotánica, inventario, sistemas agroforestales.

\section{ABSTRACT}

The study was carried out in the Middle Basin of the Río Guayas, Ecuador, in the area corresponding to the cantons Mocache and Palenque. The inventory of the species was realized by participative journeys. The species were classified according to the Cronquist Filogenetics System. The data about the use of the species were obtained by chats and non formal interviews. It was determined that the farmers from the Middle Basin of the Río Guayas know and they use 262 vegetable species that belong to 67 families and 164 genuses. The most common vegetable families were: Anacardiaceae, Papilonaceae, Solanaceae, Rutaceae, Musaceae and Lamiaceae, with more than ten species each one. Some 21 types of uses were documented, of which the most important are: nutritious, medicinal and construction, which demonstrates the concern of the families to satisfy their basic necessities. Most of the used species are trees, followed by grasses and bushes. Among the species of major importance for the multiple uses and products that are obtained are: Persea americana Mill. (aguacate), Artocarpus altilis Fosberg (fruta de pan), Musa sp. (plátano), Manguifera sp. (mango), Phythelephas aequatoriales Spruce (cade), Inga edulis Matius (guaba de bejuco), between others.

Key words: Traditional use, ethnobotanic, inventory, agroforestry systems.

\section{INTRODUCCIÓN}

Los sistemas agroforestales constituyen una fuente importante de productos madereros y además conservan una gran variedad de especies agrícolas, hortícolas, ornamentales y medicinales. Algunos de los campesinos asentados en el Litoral ecuatoriano, muy rico en biodiversidad (Neill, 1999), conservan sus huertos tradicionales mixtos con base en el cultivo de cacao (Torres y Alava, 1995), lo que hace suponer que poseen conocimientos tradicionales acerca del uso y manejo de las especies. El sistema de producción agrícola tradicional, "sorprende

Recibido: Septiembre, 2007. Aceptado: Febrero, 2008 Publicado como ARTÍCULO en Ciencia y Tecnología 1: 65-71. 2008. por su alta adaptación ecológica, con formas de aprovechamiento sostenido y diversificado, sin deterioro biológico y edáfico apreciable" (Nations y Nigh, 1980; Marion, 1991).

La información taxonómica y etnobotánica detallada, permitirá plantear alternativas de aprovechamiento que incidan favorablemente en la economía campesina (Ruiz y Jeffrey, 1993). Además en los últimos años se ha prestado especial atención a la utilización de la información etnobotánica para la selección de plantas en la búsqueda de compuestos con actividad biológica (Revelo, 1994). Algunos investigadores evidencian la importancia de utilizar información etnobotánica para 
este fin (Akerele, 1993; Zuluaga, 1994; Bermúdez et al., 2005).

En muchos países en desarrollo ha ocurrido una pérdida importante del conocimiento tradicional sobre el uso de las plantas medicinales y de otras plantas útiles (Caniago y Siebert, 1998; Benz et al., 2000; Katewa et al., 2004). Conociendo esta realidad la Unidad de Investigación Científica y Tecnológica de la Universidad Técnica Estatal de Quevedo, pretende generar información que permita conservar y difundir el conocimiento campesino, que a través de la historia se ha mantenido de generación en generación y que paulatinamente se está perdiendo por la fuerte influencia tecnológica. Es importante preservar esta herencia cultural y registrar la información sobre las especies útiles, que podrían ser relevantes para el desarrollo de nuevos medicamentos y otros beneficios para la humanidad, contribuyendo al mismo tiempo a proteger la biodiversidad (Katewa et al., 2004). El objetivo de esta investigación fue identificar y clasificar taxonómicamente las especies de los sistemas agroforestales tradicionales y determinar el conocimiento local sobre los usos de las mismas.

\section{MATERIALES Y MÉTODOS}

El estudio se realizó en la Cuenca Media del Río Guayas, en el área correspondiente a los cantones Mocache y Palenque. Entre las coordenadas geográficas: $79^{\circ} 25^{\prime}$ hasta $79^{\circ} 45^{\prime}$ de longitud oeste y $1^{\circ} 2^{\prime}$ hasta $1^{\circ} 20^{\prime}$ de latitud sur; con altitud de $73 \mathrm{msnm}$. Esta área pertenece a la formación ecológica bosque húmedo-tropical (Holdrige, 1987).

La población estuvo constituida por 560 familias campesinas, que habitan un área de 5000 hectáreas aproximadamente. Se procedió a realizar un recorrido general por la zona para seleccionar 10 fincas representativas. Se realizó un Diagnóstico Participativo de Sistemas Rurales (DPSR), descrito por Chambers (1992), que consistió en la utilización de las siguientes técnicas participativas: entrevistas semiestructuradas, recorridos participativos y reuniones con las familias.

Los predios estudiados se seleccionaron de acuerdo a los siguientes criterios (Valarezo, 1999): Ser representativos de las condiciones naturales y sistemas de producción del sector, constituir una unidad productiva en la que viva el agricultor con su familia, que los propietarios sean de avanzada edad por el cúmulo de conocimientos que poseen, y alto grado de interés y entusiasmo por participar en el proyecto.

Se elaboró un inventario de las especies útiles presentes en los sistemas agroforestales y se registró la percepción campesina sobre la utilidad de las mismas. Las especies se clasificaron según el sistema filogenético de Cronquist, citado por Cerón (1993). Además se clasificó a las especies según el hábito de crecimiento en: árboles, arbustos, hierbas, sub-arbustos, palmas, trepadoras, rastreras y bejucos.

\section{RESULTADOS Y DISCUSIÓN}

Se encontró un total de 262 especies útiles en la zona bajo estudio que corresponden a 67 familias y 164 géneros (Anexo 1), coincidiendo con lo reportado por Cerón (1996) quien manifiesta que el número de plantas útiles de un grupo étnico en Latinoamérica oscila entre 120 a 650 especies. El número de especies útiles encontradas en esta área es mayor a otros estudios realizados en el Ecuador (Cerón et al., 1994). Similarmente Traversa et al. (2000) y Trujillo et al. (2003) registraron 244 y 283 especies útiles en comunidades de México y Costa Rica, respectivamente. Por su parte, Levasseur et al. (2000) reportan el uso de 164 especies de plantas en los sistemas agroforestales tradicionales de una comunidad Maya.

Las familias más representativas son: Anacardiaceae, Papilonaceae, Solanaceae, Rutaceae, Lamiaceae, Musaceae y Moraceae (Figura 1).

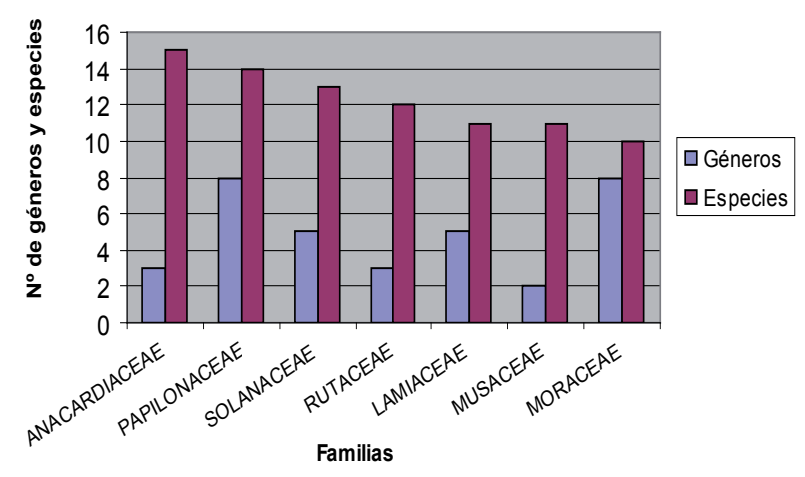

Figura 1. Familias representativas a nivel de género y especies

Se encontró que las especies de árboles fueron más abundantes con un número de 111 (42.36\%) seguidos por las plantas herbáceas con 51 especies (19.46\%) y 50 arbustos (19.08\%) (Cuadro 1). Traversa et al. (2000) también encontró una mayor cantidad de árboles (31.14\%), hierbas y arbustos (62.62\%) en los huertos caseros de Oaxaca, México. De igual manera BentesGama et al. (1999) registró un mayor número de árboles (41\%) y un $38 \%$ de especies herbáceas en los huertos caseros de la comunidad de Villa Cuera en Brasil. Por su lado, Levy et al. (2002) registraron los siguientes resultados: árboles $(35.3 \%)$, bejucos $(13.4 \%)$, arbustos (11.6\%), hierbas (10.1\%) en la comunidad lacandona, Chiapas, México. 
Cuadro 1. Hábito de crecimiento de las especies vegetales utilizadas en la Cuenca Media del Río Guayas

\begin{tabular}{ccc}
\hline Habito & $\mathrm{N}^{\mathrm{o}}$ especies & Porcentaje \\
\hline Árboles & 111 & 42.36 \\
Hierbas & 51 & 19.46 \\
Arbustos & 50 & 19.08 \\
Sub-arbustos & 20 & 7.63 \\
Rastreras & 12 & 4.58 \\
Trepadoras & 9 & 3.43 \\
Palmas & 8 & 3.05 \\
Bejuco & 1 & 0.38 \\
\hline Total & 262 & 100.00 \\
\hline
\end{tabular}

Las 262 especies encontradas se clasificaron en 21 formas de usos (Figura 2). Similarmente Levy et al., (2002) registraron 23 categorías de uso de la flora en una comunidad campesina de México. Se registró un total de 416 usos; teniendo cada especie un promedio de 1.6 formas de uso. Este valor es equivalente al reportado por Durán (1999) quien encontró 1.7 formas de uso por especie en Nahá, México. Por su parte Levy et al. (2002) señala un promedio de 1.3 para la comunidad lacandona en México. Los usos más importantes que se registraron en el presente estudio fueron: alimenticio, medicinal, maderables, leña, carbón y construcción. Bentes-Gama et al. (1999), Durán (1999), Levasseur et al. (2000), Traversa et al. (2000), Mora y Acosta (2001), Levy et al. (2002) y Trujillo et al. (2003) coinciden en que la mayoría de las especies utilizadas por distintos grupos étnicos se destina a satisfacer las necesidades básicas, citando entre las principales los usos alimenticio, medicinal y maderable. Según Toledo (1987) este patrón se repite y parece constituir una regla en los diferentes estudios etnobotánicos realizados en diferentes zonas de Latinoamérica. Los campesinos asentados en la Cuenca Media del Río Guayas, Ecuador, son originarios de la Costa, por lo que tienen un conocimiento histórico de la región, lo que ha permitido que se desarrolle un proceso de acumulación de conocimientos propios para el manejo de los sistemas agroforestales. Esto evidencia la estrecha relación de las comunidades con sus recursos naturales (Castro et al., s. f.).

En la alimentación se utilizan 104 especies (Figura 2), lo que indica que la prioridad de los campesinos es la seguridad alimentaria. Coincidiendo con Traversa et al. (2000) quienes señalan que el objetivo principal de los huertos tradicionales es la búsqueda de la autosuficiencia alimenticia y que la frecuencia de especies por uso es el indicador más adecuado para conocer el objetivo principal de un huerto. Similarmente Trujillo et al. (2003) registraron 103 especies comestibles en un estudio realizado en fincas cacaoteras indígenas en Costa Rica. Este número es superior a lo registrado por Castro et al. (s. f.) quienes señalan que tres comunidades rurales en Colombia utilizan tradicionalmente 70 especies vegetales para la alimentación. Por otro lado, en la comunidad lacandona de México los indígenas utilizan en la alimentación 96 especies vegetales (Levy et al., 2002).
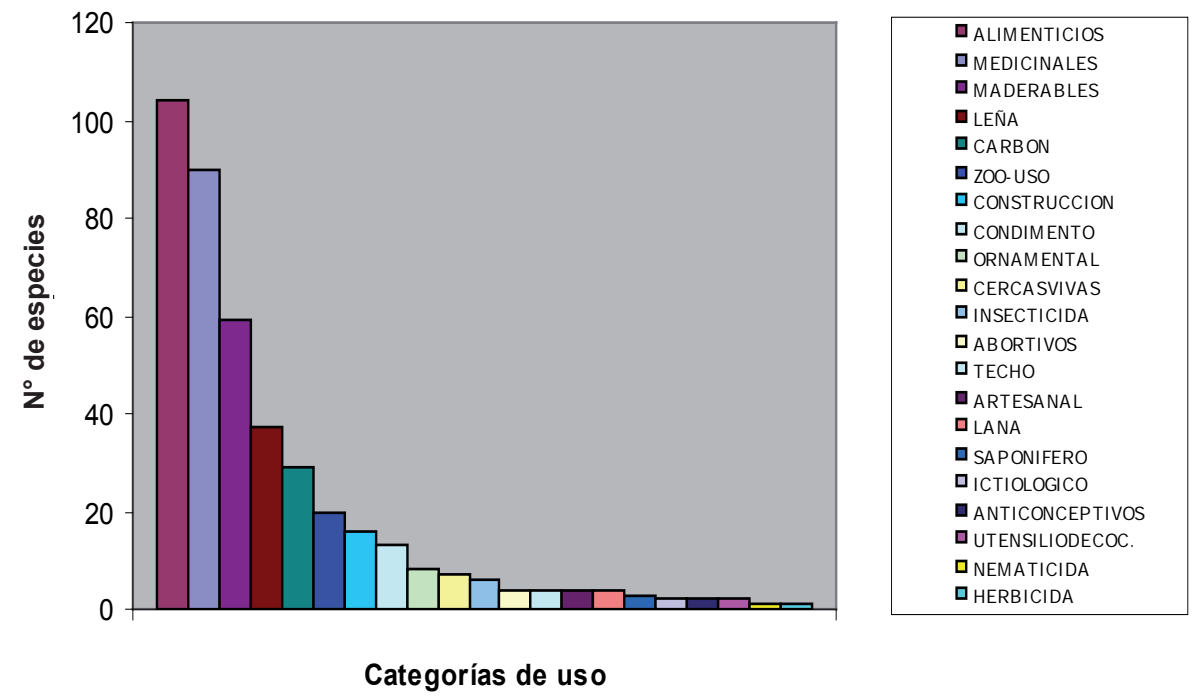

Figura 1. Número de especies según su uso tradicional en la Cuenca Media del Río Guayas 
Tradicionalmente, los campesinos han sido los abastecedores de alimentos para las poblaciones urbanas (Kolmans y Vásquez, 1996). En sus fincas se encuentra una gran cantidad de productos que dispone la población ecuatoriana para su alimentación. Las especies de consumo masivo que se producen en estas fincas son: arroz (Oriza sativa L.), banano (Musa sp.), naranja (Citrus $s p$.), mango (Manguifera sp.), yuca (Manihot esculenta Crantz), maní (Arachis hipogea L.), habichuela (Vicia $s p$.), verduras (Phaseolus sp.), fréjol túmbez (Phaseolus vulgaris L.), fréjol de palo (Cajanus cajan (L.) Millsp.), entre otras (Cuadro 2), las cuales se encontraban presente en las 10 fincas estudiadas. Esto coincide con lo citado por Calispa (1999), quien además señala que estas especies han sido conservadas y mejoradas por los propios campesinos.

Los productores del sector Mocache-Palenque de la Cuenca Media del Río Guayas conocen el uso medicinal de 90 especies (Figura 2) que utilizan para sanar diversas enfermedades como: tos, colesterol, inflamaciones, hemorragias, molestias hepáticas y renales, diarrea; entre otras (Cuadro 3). Este número es superior al registrado por Traversa et al. (2000) en los huertos caseros de Zaachila, México (38 especies), y similar al utilizado por otros grupos étnicos: Trujillo et al. (2003) indican que indígenas de tres comunidades en Costa Rica utilizan 104 especies con fines medicinales. El Anacardium occidentale L., que sirve para reducir el colesterol y otras enfermedades (Cuadro 3), también es utilizado medicinalmente por la comunidad ribereña de Villa Cuera, Brasil (Bentes-Gama et al., 1999). La información etnobotánica sobre las plantas medicinales recopilada en ésta y otras investigaciones puede servir de base para estudios farmacológicos (Akerele, 1993; Revelo, 1994; Zuluaga, 1994, Bermúdez et al., 2005). Además, la mayoría de productos medicinales obtenidos científicamente han tenido como base estudios etnobotánicos, lo que demuestra que la forma correcta de estudiar las plantas medicinales es preguntar a los campesinos e indígenas (Zuluaga, 1994).

Cuadro 2. Principales especies utilizadas para la alimentación de las familias en la Cuenca Media del río Guayas

\begin{tabular}{|c|c|c|c|}
\hline Nombre común & Nombre científico & Familia & Parte utilizada $^{\dagger}$ \\
\hline Arroz & Oriza sativa $\mathrm{L}$. & Poaceae & $\mathrm{S}$ \\
\hline Banano & Musa paradisiaca $\mathrm{L}$. & Musaceae & $\mathrm{F}$ \\
\hline Naranja & Citrus aurantifolia (Christm.) Swingle & Rutaceae & $\mathrm{F}$ \\
\hline Mango & Manguifera sp. & Anacardiaceae & $\mathrm{F}$ \\
\hline Papaya & Carica papaya $\mathrm{L}$. & Caricaceae & $\mathrm{F}$ \\
\hline Yuca & Manihot esculenta Crantz & Euphorbiaceae & $\mathrm{R}$ \\
\hline Maní & Arachis hipogea L. & Papilonaceae & S \\
\hline Habichuela & Vicia sp. & Papilonaceae & $\mathrm{S}$ \\
\hline Verduras & Phaseolus sp. & Papilonaceae & V \\
\hline Fréjol túmbez & Phaseolus vulgaris L. & Papilonaceae & S \\
\hline Aguacate & Persea americana Mill. & Lauraceae & $\mathrm{F}$ \\
\hline Fréjol de palo & Cajanus cajan (L.) Millsp. & Papilonaceae & $\mathrm{S}$ \\
\hline Maíz & Zea mays $\mathrm{L}$. & Poaceae & $\mathrm{S}$ \\
\hline Café & Coffea arabica $\mathrm{L}$. & Rubiaceae & S \\
\hline Plátano & Musa sp. & Musaceae & $\mathrm{F}$ \\
\hline Fruta de pan & Artocarpus altilis (Parkinson) Fosberg & Moraceae & $\mathrm{S}$ \\
\hline Piña & Ananas comusus (L.) Merril & Bromeliaceae & $\mathrm{F}$ \\
\hline Zapote & Matisia cordata Bonpl. & Bombacaceae & $\mathrm{F}$ \\
\hline
\end{tabular}

${ }^{\dagger} \mathrm{F}=$ fruto, $\mathrm{S}=$ semilla, $\mathrm{R}=$ rizoma, $\mathrm{V}=$ verduras 
Cuadro 3. Principales especies medicinales de la Cuenca Media del río Guayas

\begin{tabular}{|c|c|c|c|}
\hline Nombre común & Nombre científico & Virtud atribuida ${ }^{2}$ & Parte utilizada \\
\hline $\begin{array}{l}\text { Llantén } \\
\text { Zorrila }\end{array}$ & $\begin{array}{l}\text { Plantago major, L. } \\
\text { Capparis cordata. Ruiz \& Pav. }\end{array}$ & $\mathrm{C} 1$ & $\begin{array}{l}\text { Hojas } \\
\text { Hojas }\end{array}$ \\
\hline $\begin{array}{l}\text { Marañón comestible } \\
\text { Achocha de monte }\end{array}$ & $\begin{array}{l}\text { Anacardium occidentale, } \mathrm{L} . \\
\text { Ciclanthera sp. }\end{array}$ & $\mathrm{C} 2$ & $\begin{array}{l}\text { Hojas } \\
\text { Hojas }\end{array}$ \\
\hline $\begin{array}{l}\text { Mastrante } \\
\text { Guayaba }\end{array}$ & $\begin{array}{l}\text { Lepechinia mutica (Benth.) Epling } \\
\text { Psidium guajava, L. }\end{array}$ & $\mathrm{D}$ & Fruto y hojas \\
\hline $\begin{array}{l}\text { Escancel } \\
\text { Espanto }\end{array}$ & $\begin{array}{l}\text { Aerba sp } \\
\text { Peperomia sp. }\end{array}$ & $\mathrm{H}$ & Hojas y cogollos \\
\hline $\begin{array}{l}\text { Guanábana } \\
\text { Bototillo }\end{array}$ & $\begin{array}{l}\text { Annona muricata, } \mathrm{L} \text {. } \\
\text { Cochlospermun vitifolium, Mark E. Olson. }\end{array}$ & I & $\begin{array}{l}\text { Hojas } \\
\text { Hojas }\end{array}$ \\
\hline $\begin{array}{l}\text { Berdolaga } \\
\text { Albahaca olorosa }\end{array}$ & $\begin{array}{l}\text { Portulacca oleraceae L. } \\
\text { Salvia sp. }\end{array}$ & A & $\begin{array}{l}\text { Hojas y cogollos } \\
\text { Semilla }\end{array}$ \\
\hline $\begin{array}{l}\text { Paico } \\
\text { Papaya }\end{array}$ & $\begin{array}{l}\text { Chenopodium ambrosioides } \mathrm{L} \text {. } \\
\text { Carica papaya } \mathrm{L} .\end{array}$ & $\mathrm{L}$ & Semilla \\
\hline $\begin{array}{l}\text { Torojil } \\
\text { Hierba luisa }\end{array}$ & $\begin{array}{l}\text { Mellisa officinalis, L. } \\
\text { Cymbopogon citratos (DC) Staff. }\end{array}$ & $\mathrm{N}$ & Hojas \\
\hline $\begin{array}{l}\text { Zaragoza } \\
\text { Verbena }\end{array}$ & $\begin{array}{l}\text { Aristolochia pilosa Kunth } \\
\text { Verbena litorales } \mathrm{L} .\end{array}$ & $\mathrm{P}$ & $\begin{array}{l}\text { Tallo } \\
\text { Hojas }\end{array}$ \\
\hline $\begin{array}{l}\text { Sábila } \\
\text { Diente de león }\end{array}$ & $\begin{array}{l}\text { Aloe vera }(\mathrm{L} .) \text { Burm. f. } \\
\text { Taraxacum officinale F.H. Wigg. }\end{array}$ & $\mathrm{R}$ & $\begin{array}{l}\text { Hojas } \\
\text { Hojas }\end{array}$ \\
\hline $\begin{array}{l}\text { Rosa de muerto } \\
\text { Caña fístula }\end{array}$ & $\begin{array}{l}\text { Caléndula officinalis } \mathrm{L} . \\
\text { Cassia fistula } \mathrm{L}\end{array}$ & $\mathrm{T}$ & $\begin{array}{l}\text { Flores } \\
\text { Fruto }\end{array}$ \\
\hline $\begin{array}{l}\text { Altamisa } \\
\text { Aromo }\end{array}$ & $\begin{array}{l}\text { Ambrosia arborescens, Lam } \\
\text { Acacia rorudiana, Crhistoph }\end{array}$ & A2 & $\begin{array}{l}\text { Hojas } \\
\text { Raíces }\end{array}$ \\
\hline
\end{tabular}

${ }^{\dagger} \mathrm{Cl}=$ anticanceroso $\mathrm{I}=$ antiinflamatorio, $\mathrm{C} 2=$ reduce el colesterol, $\mathrm{D}=$ antidiarreico, $\mathrm{H}=$ antihemorrágico, $\mathrm{A}=$ afecciones oculares, $\mathrm{L}=$ eliminar lombrices, $\mathrm{N}=$ controlar nervios, $\mathrm{P}=$ antipalúdico, $\mathrm{R}=$ aliviar problemas renales, $\mathrm{T}=$ antigripal, expectorante, $\mathrm{A} 2=$ abortivo

\section{CONCLUSIONES}

Los habitantes de la Cuenca Media del Río Guayas, sector Mocache-Palenque utilizan 262 especies vegetales; lo que comprueba que los sistemas agroforestales de este sector poseen una gran diversidad vegetal. Las huertas mixtas del sector Mocache-Palenque de la Cuenca Media del Río Guayas contribuyen a la conservación in situ de los recursos fitogenéticos y representan la biodiversidad característica del Litoral ecuatoriano. El conocimiento local de los campesinos de la Cuenca Media del Río Guayas sobre el uso de las especies presentes en los sistemas agroforestales abarca aspectos relacionados a la satisfacción de sus necesidades básicas; siendo los usos alimenticios (104 especies) y medicinal (90 especies) los más importantes.

\section{LITERATURA CITADA}

Akerele, O. 1993. Las plantas medicinales: un tesoro que no debemos desperdiciar. Foro Mundial de la Salud. 14: 390- 395.

Bentes-Gama, M., Gama, J. e Tourinho, M. 1999. Huertos caseros en la comunidad ribereña de Villa Cuera, en el Municipio de Bragança en el Noroeste Paraense.

Benz, F., Cevallos, J., Santana, F., Rosales, J. and Graff, M. 2000. Losing knowledge about plant use in the Sierra at the Manantlan Biosphere Reserve, Mexico. Econ. Bot. 54: 183-191.

Bermúdez, A., Oliveira-Miranda, M., Velázquez, D. 2005. La Investigación etnobotánica sobre plantas medicinales: Una revisión de sus objetivos $\mathrm{y}$ enfoques actuales. Interciencia. 30 (8): 453-459. Consultado el 26 Septiembre del 2007. Disponible en: http: /www2.scielo. org.ve/scielo.php?script $=$ sci_arttext\&pid $=\mathrm{S} 0$ $37818442005000800005 \& \operatorname{lng}=\mathrm{es} \& \mathrm{nrm}=\mathrm{iso}>$.

Caniago, S. and Siebert, S. 1998 Medicinal plants ecology, knowledge and conservation in Kalimantan, 
Indonesia. Econ. Bot. 52: 229-250.

Calispa, F. 1999 Por la defensa de la agricultura campesina sana. Mimeografiado.

Castro, A., Abadía, D., Pino, N. (s f). Plantas silvestres alimenticias de uso tradicional en las comunidades de Pavurita, San José de Purre y Guayabal. Municipio de Quibdo, Chocó. Colombia. Disponible en productosnaturales_utch@starmedia.com. Consultado el 17 de septiembre del 2007.

Cerón, C. 1993. Manual de botánica ecuatoriana. Universidad Central del Ecuador. Quito, Ecuador. $191 \mathrm{p}$.

Cerón, C. 1996. Etnobotánica del Ecuador. Ed. AbyaYala. Quito- Ecuador. 195 p.

Cerón, C., Montalvo, C., Umenda, J. y Chica, E. 1994. Etnobotánica y notas sobre diversidad vegetal en la comunidad Cofán de Sinangüe; Sucumbíos, Ecuador. Ecociencia. Quito.

Chambers, R. 1992. Diagnóstico Rurales Participativos: Pasado, presente y Futuro. En revista: Bosques, árboles y comunidades rurales. $N^{\circ}$. 15/16. Colombia. C.A.

Durán, F. 1999. Estructura y etnobotánica de la selva alta perennifolia de Nahá, Chiapas. Tesis. Facultad de Ciencias. Universidad Nacional Autónoma de México. México, D.F. 150 p.

Holdrige, 1987. Ecología basada en zonas de vida. IICA. San José, Costa Rica. 216 p.

Katewa, S., Chaudhary, B. and Jain, A. 2004. Folk herbal medicines from tribal area of Rajastan, India. J. Ethnopharmacol. 92: 41-46.

Kolmans, E. y Vázquez, D. 1996. Manual de Agricultura Ecológica: Una introducción a los principios básicos y su aplicación MELA-SIMA, Managua - Nicaragua, 222p.

Levasseur, V., Olivier, A. y Somarriba, E. 2000. Sistemas agroforestales tradicionales en una comunidad maya en Belice. Agroforestería de las Américas. 7 (26): 21-23.

Levy, T., Israel, S., Aguirre, R., Martínez, M. y Durán, A. 2002. Caracterización del uso tradicional de la flora espontánea en la comunidad lacandona de Lacanhá, Chiapas, México. INCI. [Online]. Interciencia. 27 (10): 512-520.

Marion, M. 1991. Los hombres de la selva, un estudio de tecnología cultural en medio selvático. Instituto Nacional de Antropología e Historia. México, D.F. $287 \mathrm{p}$.

Mora, J. y Acosta, L., 2001. Uso y clasificación y manejo de la vegetación asociada al cultivo de café (Coffea arabica) desde la percepción campesina en Costa Rica. Agroforestería de las Américas. 8 (22): 20- 27.

Nations, J. and Nigh, R. 1980. The evolutionary poten- tial of Lacandon Maya sustained-yield tropical rain forest agriculture. J. Anthropological Res. 36: 1-33.

Neill, D. 1999. Geografía, Pp. 2-5. En: P.M. Jorgensen \& S. León-Yánez (eds.). Catalogue of the vascular plants of Ecuador. Monogr. Syst. Bot. Missouri Bot. Gard. 75.

Revelo, N. 1994. Valor económico, usos y métodos de extracción de sangre de drago, Croton sp, en el Alto Napo, Esmeraldas. In. Etnobotánica, valoración económica y comercialización de recursos florísticos silvestres en el Alto Napo, Ecuador. Ecociencia. Quito.

Ruiz, M. and Jeffrey, S. 1993. El extractivismo en América Latina. Programa de Conservación de Bosques de la UICN/Comisión de la Comunidad Europea. $97 \mathrm{pp}$.

Toledo, V. 1987 La etnobotánica en Latinoamérica: vicisitudes, contextos, desafíos. Memorias del Simposio de Etnobotánica: IV Congreso Latinoamericano de Botánica. Instituto Colombiano para el Fomento de la Educación Superior. pp 13-34.

Torres, E. y Álava, A., 1995. Diagnóstico agrosocioeconómico de los sistemas de producción prevalentes a nivel de pequeñas fincas en la Zona Central del Litoral ecuatoriano. Tesis de grado. Universidad Técnica Estatal de Quevedo. 124 p.

Traversa, T., Fierros, A., Gómez, M., Leyva, J. y Hernández, R. 2000. Los huertos caseros de Zaachila en Oaxaca, México. Agroforestería de las Américas. 7 (28) 12-15.

Trujillo, L., Somarriba, E. y Harvey, C. 2003. Plantas útiles en las fincas cacaoteras indígenas Bribri y Cabécar de Salamanca, Costa Rica. Agroforestería de las Américas. 10 (37): 36- 41.

Valarezo, 1999. Modelo de producción integral para fincas. Documento preparado para el seminario taller nacional sobre desarrollo integral de investigación. Impresión PREDESUR. Loja, Ec. 75 p.

Zuluaga, F. 1994. "Plantas Medicinales". In. Memorias del curso de campo sobre Biodiversidad y Recursos Genéticos Indígenas y Campesinos. Diversidad Biológica y Diálogo de saberes. Maestría en Desarrollo Sostenible de Sistemas Agrarios. Cali, Colombia. 
Anexo 1. Familias Botánicas, géneros y especies registradas en la Cuenca Media del Río Guayas

\begin{tabular}{|c|c|c|c|}
\hline No & Familia & $\mathrm{N}^{\circ}$ Géneros & $\mathrm{N}^{\circ}$ Especies \\
\hline 1 & Anacardiaceae & 3 & 15 \\
\hline 2 & Papilonaceae & 8 & 14 \\
\hline 3 & Solanaceae & 5 & 13 \\
\hline 4 & Rutaceae & 3 & 12 \\
\hline 5 & Lamiaceae & 5 & 11 \\
\hline 6 & Musaceae & 2 & 11 \\
\hline 7 & Moraceae & 10 & 10 \\
\hline 8 & Mimosaceae & 5 & 9 \\
\hline 9 & Poaceae & 6 & 9 \\
\hline 10 & Annonaceae & 4 & 8 \\
\hline 11 & Arecaceae & 5 & 8 \\
\hline 12 & Piperaceae & 2 & 7 \\
\hline 13 & Aracaceae & 4 & 6 \\
\hline 14 & Caesalpinaceae & 4 & 6 \\
\hline 15 & Cucurbitaceae & 6 & 6 \\
\hline 16 & Euphorbiaceae & 6 & 6 \\
\hline 17 & Meliaceae & 5 & 6 \\
\hline 18 & Rubiaceae & 5 & 6 \\
\hline 19 & Sterculiaceae & 3 & 5 \\
\hline 20 & Myrtaceae & 3 & 5 \\
\hline 21 & Clusaceae & 3 & 5 \\
\hline 22 & Bignonaceae & 2 & 4 \\
\hline 23 & Verbenaceae & 4 & 4 \\
\hline 24 & Sapotaceae & 3 & 4 \\
\hline 25 & Aquifoliaceae & 2 & 3 \\
\hline 26 & Bixaceae & 1 & 3 \\
\hline 27 & Bombacaceae & 3 & 3 \\
\hline 28 & Bromeliaceae & 2 & 3 \\
\hline 29 & Compositae & 3 & 3 \\
\hline 30 & Convolvulaceae & 3 & 3 \\
\hline 31 & Lauraceae & 2 & 3 \\
\hline 32 & Passifloraceae & 1 & 3 \\
\hline 33 & Sapindaceae & 3 & 3 \\
\hline 34 & Urticaceae & 2 & 2 \\
\hline 35 & Zingiberaceae & 2 & 2 \\
\hline 36 & Geraniaceae & 2 & 2 \\
\hline 37 & Boraginaceae & 1 & 2 \\
\hline 38 & Asteraceae & 2 & 2 \\
\hline 39 & Amaranthaceae & 1 & 1 \\
\hline 40 & Apiaceae & 1 & 1 \\
\hline 41 & Aristolochiaceae & 1 & 1 \\
\hline 42 & Caricaceae & 1 & 1 \\
\hline 43 & Cecropiaceae & 1 & 1 \\
\hline 44 & Cochlospermaceae & 1 & 1 \\
\hline 45 & Combretaceae & 1 & 1 \\
\hline 46 & Chenopodiaceae & 1 & 1 \\
\hline 47 & Cyclanthaceae & 1 & 1 \\
\hline 48 & Equisetaceae & 1 & 1 \\
\hline 49 & Capparidaceae & 1 & 1 \\
\hline 50 & Juglandaceae & 1 & 1 \\
\hline 51 & Liliaceae & 1 & 1 \\
\hline 52 & Lecythidaceae & 1 & 1 \\
\hline 53 & Malpighiaceae & 1 & 1 \\
\hline 54 & Malvaceae & 1 & 1 \\
\hline 55 & Marantaceae & 1 & 1 \\
\hline 56 & Myristicaceae & 1 & 1 \\
\hline 57 & Ovalidaceae & 1 & 1 \\
\hline 58 & Pedaliaceae & 1 & 1 \\
\hline 59 & Plantaginaceae & 1 & 1 \\
\hline 60 & Polygonaceae & 1 & 1 \\
\hline 61 & Rhannaceae & 1 & 1 \\
\hline 62 & Salicaceae & 1 & 1 \\
\hline 63 & Scrophulariaceae & 1 & 1 \\
\hline 64 & Simaroubaceae & 1 & 1 \\
\hline 65 & Teaceae & 1 & 1 \\
\hline 66 & Ulmaceae & 1 & 1 \\
\hline \multirow[t]{3}{*}{67} & Violaceae & 1 & 1 \\
\hline & F. indeterminada & & 6 \\
\hline & Total & 164 & 67 \\
\hline
\end{tabular}

